\title{
Development of Methods Acquiring Real Time Very High Resolution Agricultural Spatial Information Using Unmanned Aerial Vehicle
}

Basuti Bolo, Dimane Mpoeleng, Irina Zlotnikova

Botswana International University of Science and Technology, Palapye, Botswana

\begin{abstract}
There is a need for high resolution spatial information to provide quality agricultural spatial information for better monitoring and management of farm activities to increase production and sustainable agricultural economic development. The Unmanned Aerial Vehicles are able to capture very high resolution spatial data that can be transformed into useful geospatial information, databases and digital maps. However, Unmanned Aerial Vehicle methods of acquiring spatial data are yet to be developed. The objective of this study was to develop methods of acquiring real time high resolution agricultural spatial data using Unmanned Aerial Vehicle. A qualitative case study research approach, and data collection method were used to achieve the objective. A ground truth data was carried out to eliminate errors. Unmanned Aerial Vehicle data acquisition system and data processing methods were developed. These methods could be used for better farm management and reduce the cost of inputs like fertilizers.
\end{abstract}

\section{Keywords}

UAV, agricultural data, data acquisition, very high resolution data..

Bolo, B., Mpoeleng, D. and Zlotnikova, I. (2019) "Development of Methods Acquiring Real Time Very High Resolution Agricultural Spatial Information Using Unmanned Aerial Vehicle", AGRIS on-line Papers in Economics and Informatics, Vol. 11, No. 2, pp. 21-29. ISSN 1804-1930. DOI 10.7160/aol.2019.110203.

\section{Introduction}

This study aimed at developing methods of acquiring real time very high resolution agricultural spatial data using Unmanned Aerial Vehicle (UAV) to manage farm activities. Farm management when done properly could increase production and profit, and lead to an increase in agricultural economic production. The real time high resolution data and spatial information on agricultural farming is a need for agricultural land management, development and sustainable crop production. Imagery allows for observation of individual plants and patterns over the landscapes (Franklin et al., 2014; Laliberte et al., 2006). The high demands of very high resolution spatial data are advancing thus leading to the discovery of the new platforms. A new platform of the spatial data acquisition is based on Unmanned Aerial Vehicle (UAV). This new platform can provide real time very high resolution spatial data. UAV is one of the powerful equipment that can be used to obtain the earth surface images from any altitude (Tahar, 2012). High quality data can be later be transformed and used for obtaining geospatial information. Unmanned Aerial Vehicles (UAVs) have shown to be capable for agricultural Remote sensing data collection (Xiang and Tian, 2011). The agricultural spatial data had been typically captured by the manned mission known as satellites. Satellites had some limitations on providing high resolution data, and the imagery are of adequate spatial and temporal resolution.

Importance of UAV in provision of spatial data and information

Unmanned Aerial Vehicles (UAVs) play a role complementary to that of satellites and conventionally piloted aircraft (Cano et al., 2017). Although satellites are useful for global and regional data acquisition, they are limited in their ability to provide data of adequate spatial and temporal resolution (Cano at el., 2017). UAV overcome limitations of satellites by providing a flow of real time data and allowing timely information (Liaghat and Balasundram, 2010). UAV provide real time, more high quality imagery data as compared to satellite imagery data (Zinkie and Flener, 2013), (Antle et al., 2015). UAV fly in low altitude, without cloud interference and can take higher resolution pictures (Zinkie 
and Flener, 2013), (Yano et al., 2016). UAV uses the combination of simple digital photographic cameras with spectral filters designed to provide multi-spectral images in the visible and infra-red domains (Kahan, 2007). UAV provides the real time aerial photographs and information, and can produce both high and low resolution data on both small and large spatial coverage. UAV can monitor crops and unreachable areas any time anywhere. UAV bridges the gap in scale and resolution between ground observations and imagery acquired from manned aircraft and satellite sensors (Oltmanns et al., 2012). It also reduces the gap between fieldwork and low resolution data acquisition.

However the use of UAV application in agriculture is not common. There is insufficient information and methods on how to acquire the data and transform it into a useful information. This study developed methods on how to capture high resolution spatial agricultural data of a ploughed farm using UAV. The data captured was in a form of images.The methods for calculating the spatial resolutions were developed based on the images captured by an UAV.

This study used UAV to fill the gap on provision of high resolution quality spatial data and developed methods of acquiring real time very high resolution agricultural spatial data. The data acquisition system, methods of capturing the data and calculation of spatial resolution were developed and can be used by other researchers as a reference. The captured data and developed methods could be used to manage agricultural farm activities for better management and to increase production.

\section{General background}

Unmanned Aerial Vehicle (UAV) based agricultural Remote sensing systems have been developed by several researchers (Herwitz et al., 2004). UAV had been used in many industries such as in forestry, surveying and mapping, disasters and risks management and agriculture. This study focused on how the UAV technology had been used in agriculture. This section presents an account of the existing studies on the use of the UAV technology in agriculture. (Peña et al., 2013) identified weeds between the lines of a corn crop using the images captured by an UAV multispectral camera. (Greenwood et al., 2016) used Remote sensing images to obtain the data of the orange fleshed sweet potato fields in Tanzania. Quality images were produced to accurately discriminate land use methods. The Lima CIP team (Allen, 2016) used the images to monitor crop stress. In India the UAV technology was used in crop insurance by the Kisan Pilot Programme (Garg, 2016). (Nigon et al., 2015) evaluated the ability of hyperspectral remote sensing to predict nitrogen stress in potatoes (Solanum tuberosum) during two growing seasons (2010 and 2011). The spectral data were evaluated using ground based measurements of leaf nitrogen concentration. These researches had provided a basic use of UAV in agricuture. There is insufficient methods and tools of capturing managing and transforming UAV data into a useful geospatial information. This study aimed at developing advanced UAV methods of data capture of a very high resolution data for monitoring and managing agricultural farm activities and the surrounding environment.

\section{General and specific objectives}

The general objective of this study was to develop methods of acquiring real time very high resolution agricultural spatial data using Unmanned Aerial Vehicle (UAV).

The specific objectives of the study were as follows:

1. To identify methods and techniques of spatial data acquisition of real time very high resolution agricultural spatial data and information using UAV

2. To develop data acquisition methods to obtain real time very high resolution agricultural spatial data.

\section{Materials and methods}

The methods used to identify the technologies of spatial data acquisition of real time very high resolution was through literature from the past research. Two broad types of platforms can be used for the acquisition of aerial images (Zinke and Flener, 2013), the aircraft and the Unmanned Aerial Vehicles (UAV). The aircraft are expensive and not easy to control because they are manned vehicles. The UAVs are easy to pilot and can fly at very low altitudes, enabling them to deliver very high resolution imagery at relatively low cost (Zinke and Flener, 2013). This study used the easy piloted Unmanned Aerial Vehicle to achieve the objectives.

A qualitative case study research approach was used to achieve the main objective. Qualitative research is a means for exploring and understanding the meaning individuals or groups ascribe to a social or human problem (Creswell, 2004). In this study, the exploration and understanding 
was on the Unmanned Aerial Vehicle (UAV) for the development of methods of acquiring spatial data to process and transform it into useful geospatial information. The research approach involved questions and procedures; data collection and making interpretations of the meaning of the data (Creswell, 2004). These could be used to develop methods of UAV data acquisition and process. Qualitative methods rely on text and image data, had unique steps in data analysis, and draw on diverse designs (Creswell, 2004). This study relied on the agricultural information based on the text, and the Unmanned Aerial Vehicle (UAV) multi-spectral imagery to develop data acquisition and process methods.

Two farms were used to collect the agricultural farm activities qualitative spatial data. The data collected was used to developed methods of managing and transform the spatial data into useful geospatial information. The study used a DJI Phantom 4 quad-copter drone with a payload of a 12 pixel camera, focal length $(20 \mathrm{~mm})$ with an inbuilt of both Global Positioning System (GPS) and Global Navigation Satellite System (GLONASS) to collect the primary data. GPS and GLONASS when used together provide very high positioning accuracy real time data. UAV can be manually or autonomously controlled (Chao et al., 2010). The selection of manual or autonomous mode depends on the purpose of research. Manual control requires an operator to operate UAV during flight mission while autonomous requires ground crew station and operator to launch and land the UAV (Tahar, 2012). Manual control allows altitude to be changed easily and it reacts to real time information quickly. Autonomous is automatic and it is not easy to change altitude, it has to be reset again. In this study the data was captured mannual by a Unmanned Aerial Vehicle (UAV).

The spatial data was captured under a low altitude of five to $120 \mathrm{~m}$ above the ground on the studied objects or features. The data captured were unprocessed true colour digital images produced in a multi-spectral three bands (Red, Blue and Green) format. The images were shown in visual colours representing the colour seen by human eye.

After data collection, the data had to be verified for accuracy. This was done through the ground truth data approach. A ground truth data was carried out to eliminate errors. Ground truth the data involved checking to ensure that the collected data are the representative of what exist on the ground. This was done by surveying the data and the ground. These data was used to develop methods of UAV data aquisition. After the data had been captured, the spatial resolution was calculated in order to calculate the ratio (scale) of the images to the ground.

The spatial resolution is important for features identification and data analysis. The calculation of spatial resolution of UAV data had been considered to be complicated due to many factors involved (Propella, 2018). The UAV spatial resolution is referred to as Ground Spatial/Sample Distance (GSD) in aerial photography (Propella, 2018). The spatial resolution is the distance between two consecutive pixel centers measured on the ground.

The spatial resolution of the UAV spatial data (imagery) was determined by a combination of several factors such as sensor dimensions, aspect ratio, camera focal length and the height of the camera above the ground of the area where data (image) was being taken. The number of pixels on the sensor in the $\mathrm{X}$ (horizontal) and $\mathrm{Y}$ (vertical) was obtained from the properties of an image taken by UAV camera. The horizontal and the vertical fields of view or a camera were found in the manufacturer's specifications for the camera/lens. The spatial resolution was determined by the number of pixels in the sensor array and the field of view of the camera. The UAV spatial resolution was calculated using GSD calculator and by manually method .

In this study a GSD calculator developed by the Propeller was used to calculate the resolution. When calculating the UAV spatial resolution the followings camera parameters are needed; the image width (pixel), image height (pixel), sensor width $(\mathrm{mm})$, sensor height $(\mathrm{mm})$, focal length $(\mathrm{mm})$ and the flight height $(\mathrm{m})$. Another option is to use customize procedures where the parameters of the drone have to be entered manually. In this case, the parameters of the camera and the flight height must be known to produce accurate results.

The resolution is determined by the height or the focal length of the camera to produce quality data and information. The bigger spatial resolution value represents the lower spatial resolution of the data (image) with less visible details. The smaller spatial resolution value represents the higher spatial resolution of the data (image) with more visible details. A spatial resolution of $5 \mathrm{~cm}$ means that one pixel in the image represents linearly $5 \mathrm{~cm}$ on the ground $(5 * 5=25$ square 
centimeters). A spatial resolution of $5 \mathrm{~m}$ means that one pixel in the image represents linearly $5 \mathrm{~m}$ on the ground $(5 * 5=25$ square meters $)$.

The spatial resolution was also calculated manually based on the flight height, the sensor height, the image height, the focal length, the sensor width and the image width as shown in Figure 1.

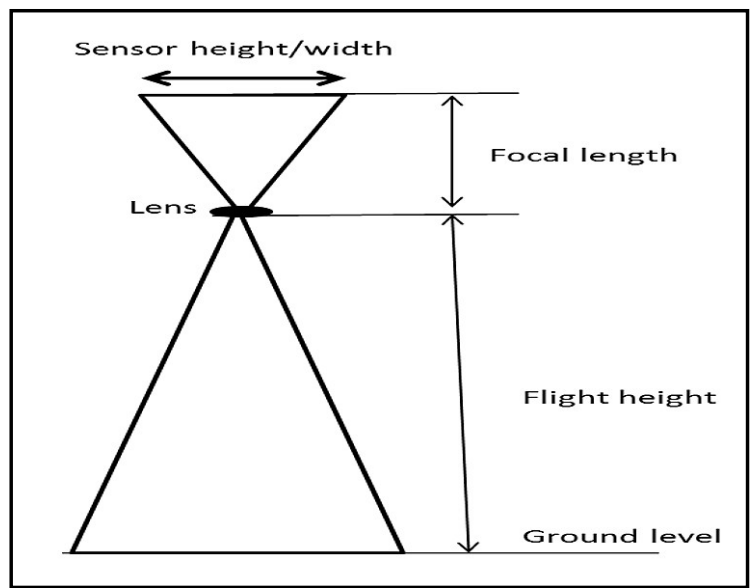

Source: Tellidis and Levin (2014), Propeller (2018)

Figure 1: Graphic representation of parameters for calculating the spatial.

The calculation was based on this manual GSD calculation formula.

$$
\begin{aligned}
& \text { GSDh }=\frac{\text { Flight Height } * \text { Sensor Height }}{\text { Focal Length } * \text { Image Height }} \\
& \text { GSDw }=\frac{\text { Flight Height } * \text { Sensor Width }}{\text { Focal Length } * \text { Image Width }}
\end{aligned}
$$

The diagram in Figure 1 is the general geometric of all aerial photographs. The parameters that were used were for the data (images) that were captured in this study.

\section{Results and discussion}

The results of this study are the methods of acquiring spatial agricultural data and information using Unmanned Aerial Vehicle (UAV). The process of methods of data acquisition of UAV is complex. Several steps have to be considered to achieve better results. This study developed an UAV data acquisition system (Figure 2) composed of six components. The system is based on the main methods of data acquisition developed during the study.

The main components for the UAV data acquisition are the people, the platform, the sensor, the earth environment (Area of Interest (AoI)), spatial data and the data process. These components are needed to acquire and provide data, and process it into useful information.

The people are the users of data and also those that operate the UAV. The motivation for data acquisition is the need by farmers, decision makers, researchers and other users. Platform is the UAV is the platform carries the payload (sensors). The sensor (camera) takes the image of the objects of interest (features on the ground) such as ploughed areas and crops. The earth environment is where the agricultural activities take

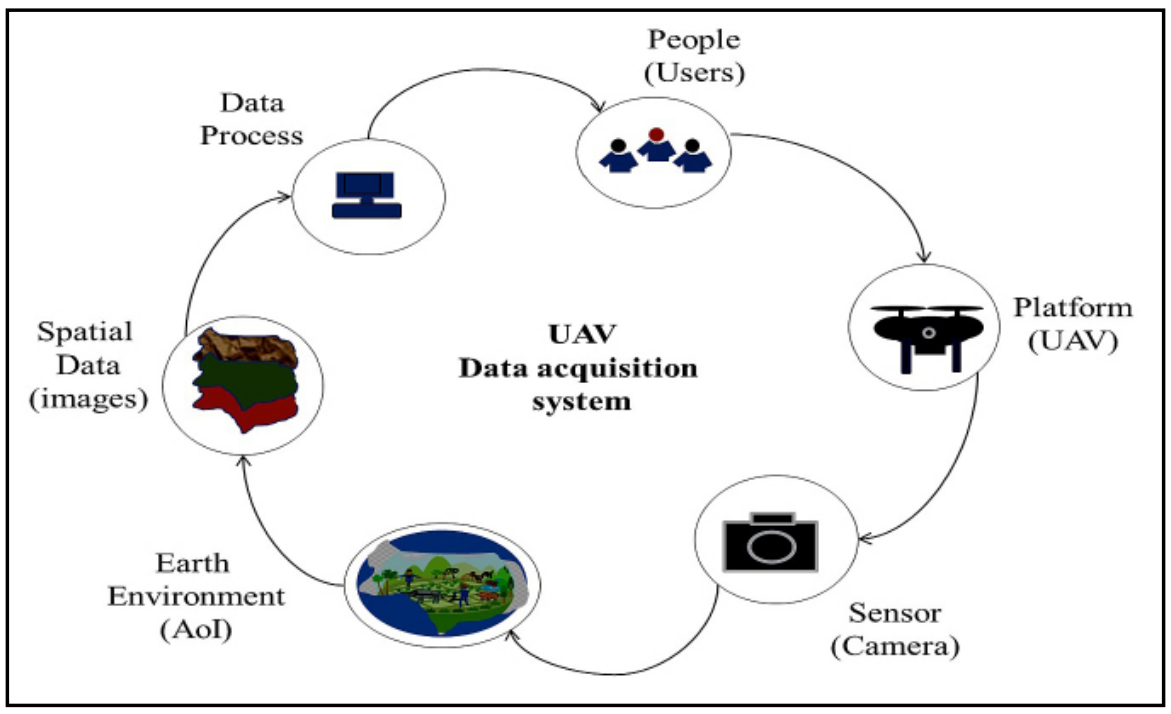

Source: own processing

Figure 2: UAV based data acquisition system. 
place. The spatial information is needed for planning purposes therefore the data is the main output of UAV data acquisition. UAV collect raw data. These data need to be processed and transformed into useful information of agriculture presented in maps for visualization. The data processing includes the methods and software. They are used to convert data into geospatial information.

The acquisition and methods comprising the data processing component of the system on Figure 2 are considered in more detail in the next two subsections, namely 1 and 2 .

\section{Procedures of data acquisition and processing}

The study identified two main procedures of data acquisition by UAV. The first procedure was developing preparation plan which included the site plan, the image acquisition plan and the flight preparation plan. The second procedure was the survey of the area of interest. Both procedures had to be completed before starting the process of data capture.

\subsection{Development of the preparation plan}

The preparation plan is needed in order to plan for the flight mission and to decide on how the data be captured. The site plan involved identification of the sites where to locate the operation of the flight. The image acquisition plan explained how the image would be captured. The flight preparation plan set parameters for the constant flying height and overlapping.

\subsection{Study area survey}

A survey of the study area was needed before UAV data acquisition. This was done to identify obstacles in the study area such as power lines, large trees and sensitive areas that could interact with the UAV. Those undesirable interactions might lead to distortion of the information and less accuracy.

\section{Capturing UAV spatial data}

An Unmanned Aerial Vehicle (UAV) was able to produce a significant amount of spatial data in a form of image scenes. The exact number of image scene depended on the size of the camera, area of interest and the height to cover the entire study area. An overlapping of images was needed to minimize the side distortions occurred when the images were being captured.

\subsection{Image overlapping}

An Unmanned Aerial Vehicle (UAV) produces many image scenes depending on the size of the camera, area of interest and the limited height to cover the entire area being studied. An overlapping of images is needed to minimize the side distortions occurred when the image is being captured. In this study an overlap of $80 \%$ forward and back, as shown in Figure 3 was done in order to avoid distortions from the edges.

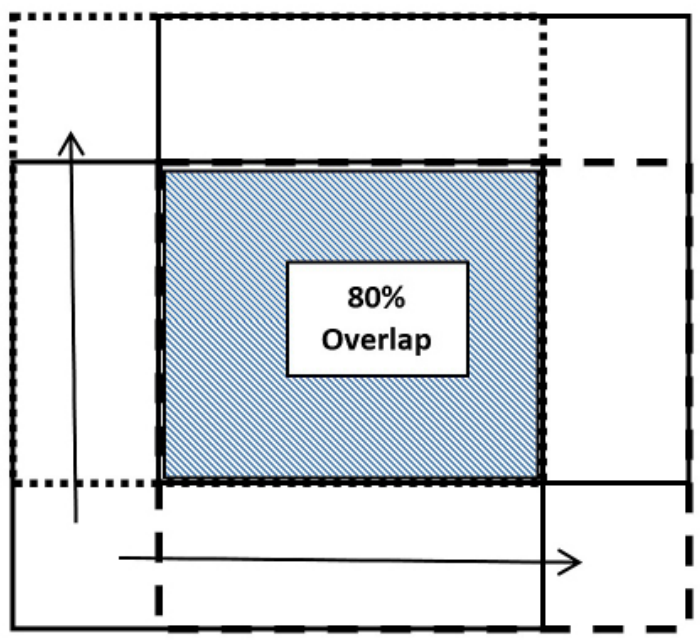

Source: own processing

Figure 3: Image overlap of $80 \%$ forward and lateral sides.

An overlapping of $80 \%$ was done also to produce a precise spatial data and information results during a transformation when the data is assigned the real world coordinates. This minimizes the error of geo-referencing of the data and data transformation error that is controlled by the quality of spatial data. In order to perform the process of image overlapping, the speed of the UAV and the altitude is balanced when capturing the images.

\subsection{The methods of spatial data capture by an UAV}

The spatial farm activities data was captured using an Unmanned Aerial Vehicle (UAV) under the manual flight control. The process is represented schematically in Figure 4. The optical axis of the camera was set perpendicular to the ground in order to capture vertical (Nadir) imagery type as shown in Figure 4. During the data capture the field of view in the real world coordinates ( $\mathrm{rw}$ ) with planar dimensions ( $\mathrm{X} \mathrm{rw}, \mathrm{Y}$ rw) is mapped to the camera sensor elements $(\mathrm{X}, \mathrm{Y})$. 


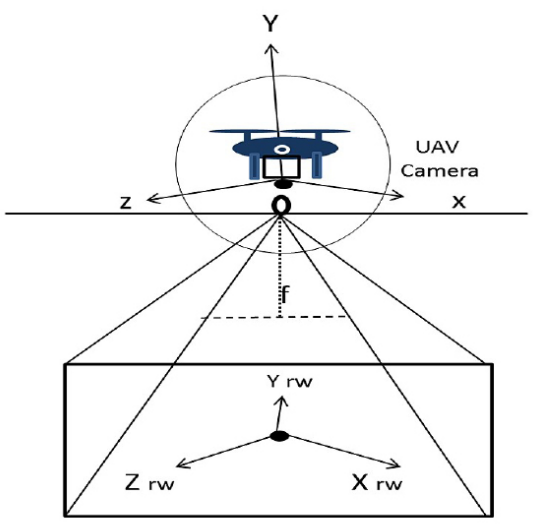

Ground Image View

Source: own processing

Figure 4: Agricultural spatial data capture by an UAV.

All the images captured by the UAV were geo-referenced to the real world coordinates. The coordinates were located at the center or focal point of the camera on the ground when the image was being captured in a vertical system.

The images were captured at different heights above the ground as shown in Figure 5, to capture views of areas of interest and to get detailed information about the farm and crop grown.

\section{Unmanned Aerial Vehicle}

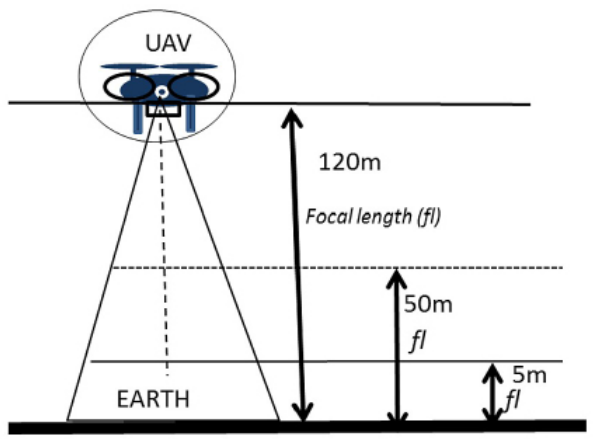

Area of Interest (Aol)

Source: own processing

Figure 5: UAV data capture in different height.
The higher height produced large ground coverage with fewer details and low resolution. The lower height produced smaller ground coverage with more details and higher resolution. The captured images were taken at the height of $120 \mathrm{~m}, 50 \mathrm{~m}$ and five meters above the ground (Figure 6). This was done to get a clear picture about the condition of crops grown.

Capturing RGB multi-spectral images at three different heights provided a clear picture of the farm activities (crops grown and how they were planted). The crops were those of beans planted in rows. The images were captured one month after the crops were planted. Using UAV, the images can be captured anytime, anywhere at certain intervals for monitoring and assessing the growth, health, and crop variability.

The images captured by an UAV, each image had a metadata (image information) attached to it. The metadata is the documentation of data. The quality of the data can easily be identified using the metatadata. The metadata stores the data quality components. The metadata included: (1) Global Positioning System (GPS) reference coordinate $(\mathrm{X}, \mathrm{Y})$ that identified the position of the image on the ground, (2) altitude (Z), (3) image bands - Red (R), Green (G) and Blue (B), (4) focal length, (5) resolution (in pixels), and (6) the date of capture. Both the data and metadata were needed for the spatial data transformation, management and sharing. The metadata of the images in Figure 5 are presented in Table 1.

The metadata is needed in the processing of data, transformation and analysis of data. Based on the images captured by an UAV, pixel size is needed when processing data using rasterization analysis. The latitude and the longitude is needed when transforming the data and georeferencing the data into a real world. The altitude is the height above sea-level. It represents the elevation or slope on the ground.
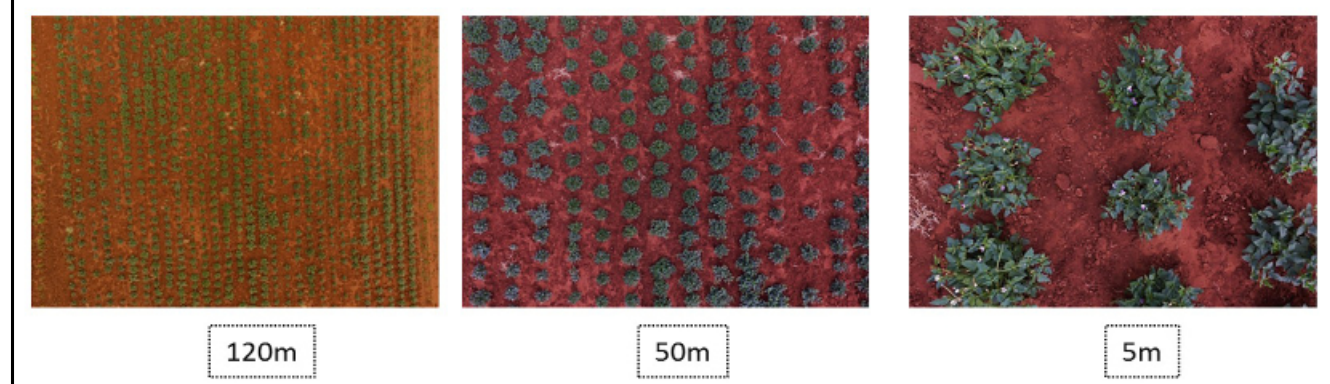

Source: own processing

Figure 6: RGB multi-spectral images showing bean crops at different heights. 


\begin{tabular}{|l|c|c|c|c|c|}
\hline Image & Multispectral & Size (pixel) & GPS Latitute & GPS Altitute & Altitute \\
\hline $120 \mathrm{~m}$ & RGB & $4000 \times 3000$ & $-22: 45: 29.523$ & $12: 53.1$ & $948.705 \mathrm{~m}$ \\
\hline $50 \mathrm{~m}$ & RGB & $4000 \times 3000$ & $-22: 45: 29.537$ & $12: 53.1$ & $934.305 \mathrm{~m}$ \\
\hline $5 \mathrm{~m}$ & RGB & $4000 \times 3000$ & $-22: 45: 29.531$ & $12: 53.1$ & $921.505 \mathrm{~m}$ \\
\hline
\end{tabular}

Source: own processing

Table 1: Metadata of UAV images.

\section{Spatial resolution of UAV data}

In this study the spatial resolution of the images were calculated using the Ground Spatial Distance (GSD) calculator and the results are shown in Table 2.

\begin{tabular}{|l|c|c|}
\hline Image Flight height & Spatial resolution & Rounded-off \\
\hline $120 \mathrm{~m}$ & $4.63 \mathrm{~cm} / \mathrm{px}$ & $5 \mathrm{~cm} / \mathrm{px}$ \\
\hline $50 \mathrm{~m}$ & $1.93 \mathrm{~cm} / \mathrm{px}$ & $2 \mathrm{~cm} / \mathrm{px}$ \\
\hline $5 \mathrm{~m}$ & $0.19 \mathrm{~cm} / \mathrm{px}$ & $0.2 \mathrm{~cm} / \mathrm{px}$ \\
\hline
\end{tabular}

Source: own processing

Table 2: GSD calculator spatial resolution results.

The results presented in Table 1 show the resolution of the image with a flight height of $120 \mathrm{~m}$ $4.63 \mathrm{~cm} / \mathrm{px}$, image with flight height of $50 \mathrm{~m}$ $(1.93 \mathrm{~cm} / \mathrm{px})$ and the image with a flight height of $5 \mathrm{~m}(0.19 \mathrm{~cm} / \mathrm{px})$.

The spatial resolution was also calculated manually based on the flight height, the sensor height, the image height, the focal length, the sensor width and the image width. The parameters that were used were for the data (images) that were captured in this study. The calculation was based on the manual GSD calculation formula below:

$$
\begin{aligned}
& \text { GSDh }=\frac{\text { Flight Height } * \text { Sensor Height }}{\text { Focal Length } * \text { Image Height }} \\
& \text { GSDw }=\frac{\text { Flight Height } * \text { Sensor Width }}{\text { Focal Length } * \text { Image Width }}
\end{aligned}
$$

To calculate the spatial resolution (GSD) for 120 $\mathrm{m}$ above the ground using manual calculation and the camera parameters in Table 3.

\begin{tabular}{|l|c|}
\hline Item & Parameters \\
\hline Image Width & 3000 pixel \\
\hline Image height & 4000 pixel \\
\hline Sensor Width & $6.17 \mathrm{~mm}$ \\
\hline Sensor Height & $4.55 \mathrm{~mm}$ \\
\hline Focal length & $4 \mathrm{~mm}$ \\
\hline
\end{tabular}

Source: own processing

Table 3: Camera parameters.
Before starting the calculations, the values of the measurements must be converted into $\mathrm{cm}$. The calculation for $120 \mathrm{~m}$ flight height was as follows;

$$
\begin{aligned}
& G S D h=\frac{12000 * 0.455}{0.4 * 4000}=3.41 \mathrm{~cm} / \text { pixel } \\
& G S D w=\frac{12000 * 0.617}{0.4 * 3000}=6.17 \mathrm{~cm} / \text { pixel }
\end{aligned}
$$

The worst case scenario in this case was the one that was used because the projected pixels onto the ground are not perfectly square. Therefore when spatial resolution is being calculated, the greatest (worst) is the one used (Propeller, 2018). The spatial resolution was $6.17 \mathrm{~cm} /$ pixel.

There was difference between the spatial resolutions of manual calculated and the one calculated by GSD calculator. The resolution calculated by a GSD calculator for a flight height of $120 \mathrm{~m}$ in this study was $4.63 \mathrm{~cm} /$ pixel while the one calculated manually was $6.17 \mathrm{~cm} /$ pixel. There was a difference of $1.17 \mathrm{~cm} /$ pixel. Based on the resolution calculated mannally, where the worst scenario was being considered, the results could be improved by combinng the two results $(\mathrm{GSDh}+\mathrm{GSDw})$, averaging them and taking the average as the final resolution result. Thus;

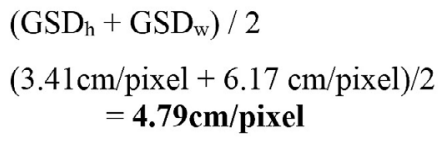

The average results were close to the one calculated by a GSD calculator which was $4.63 \mathrm{~cm} /$ pixel. Therefore the manual calculated method can be improved by adding the two results $(\mathrm{GSDh}+\mathrm{GSDw})$ then taking the average as the final result to improve accuracy.

\section{Conclusion}

The study has achieved the objectives and proved the application of Unmanned Aerial Vehicle (UAV) technologies in managing farm geographic spatial information. The study has shown the effectiveness 
of the UAV in managing agricultural crop farm activities. The study availed precise geospatial information system methods and information needed by farmers and the government to plan and forecast agricultural crop production. The researchers could use it as a reference, and advanced the methods and data acquisition system that have been developed.

During the study when capturing agricultural information, the study identified six main components of UAV data acquisition and developed an UAV data acquisition system. The system was used to collect and store UAV raw data. The system can also be improved by advancing it with other data processing tools and methods for other use. This study has improved the UAV methods of data acquisition and processing. These methods can be used to produced precise agricultural geospatial information frameworks.

The spatial data that was collected at different heights of $120 \mathrm{~m}, 50 \mathrm{~m}$, and $5 \mathrm{~m}$ using DJI Phantom 4 with 12 pixels had proved the capability of an UAV that it can be adjusted to any height during data collection any time.

The UAV techniques have proved to fill the gap between other aerial and satellite techniques with accuracies and high resolutions of a centimeter, that had been also identified by other researchers such as (Oltmanns et al, 2012), (Zinke and Flener, 2013). The data collected in this study was used to calculate the spatial resolution of the images which proved to be high at $\mathrm{cm} /$ pixel as follows; a flight height of $120 \mathrm{~m}$ above the ground, the spatial resolution was $4.63 \mathrm{~cm} / \mathrm{pixel}$ ), flight height of $50 \mathrm{~m}$ was $1.93 \mathrm{~cm} /$ pixel and, a flight height of $5 \mathrm{~m}$ was $0.19 \mathrm{~cm} /$ pixel.

From the literature methods for calculating the spatial resolution of UAV were identified and used to calculate the spatial resolution of the spatial data that was captured in this study. The methods were used in order to test their effectiveness and use them as a reference. The methods that were used and found to be effect are the GSD Propeller calculator and the Manual GSD calculation formula (Propeller, 2018).

The study has availed methods of acquiring real time very high resolution agricultural spatial data using Unmanned Aerial Vehicle (UAV). The methods that were developed and identified in this study can be used to capture the high resolution spatial data for the agricultural crop production. These data can be used to manage farm activities and reduce costs.

\section{Corresponding authors}

Basuti Bolo

Botswana International University of Science and Technology

Private Bag 16, Palapye, Botswana

Phone: +267- 72415845, Fax: +267-493 1002,Email: Basutibolo@gmail.com

Orcid. No. 0000-0003-1723-5108

\section{References}

[1] Allen, W. (2016) "Drones detect crop stresses more effectively", ÏCT Update 'Drones for Agriculture', No 82. [Online]. Available: http://ictupdate.cta.int/2016/04/21/drones-detect-crop-stresses-moreeffectively/ [Accessed: 15 Sept. 2018].

[2] Antle, J. M., Jones, J. W. and Rosenzweig, C. (2015) "Towards a New Generation of Agricultural System Models, Data, and Knowledge Products: Introduction". in: Towards a New Generation of Agricultural System Models, Data, and Knowledge Products, pp. 1-8. [Online]. Available: http://www.agmip.org/wp-content/uploads/2015/04/Towards-a-New-Generation-of-Ag-SystemsComplete.pdf [Accessed: 10 Aug 2018].

[3] Cano, R., Horton, R., Liljegren, H and Bulanon, D. M. (2017): "Comparison of Small Unmanned Aerial Vehicles Performance Using Image Processing", Journal of Imaging, Vol. 3, No. 1, 4 p. ISSN 2313-433X. DOI 10.3390/jimaging3010004.

[4] Chao, H. Y., Cao, Y. C and Chen, Y. Q. (2010) "Autopilots for Small Unmanned Aerial Vehicles: A Survey", International Journal of Control, Automation and Systems, Vol. 8, No. 1, pp. 36-44. E-ISSN 2005-4092, ISSN 1598-6446. DOI 10.1007/s12555-010-0105-z.

[5] Creswell, J. W. (2014) "Research design: qualitative, quantitative, and mixed methods approaches", $4^{\text {th }}$ ed., SAGE Publications. ISBN-13 978-1452226101, ISBN-10 1452226105. 
[6] Franklin, P., Pearlstine, L., Dewitt, B., Smith, S., Watts, A., Ifju, P. (2014) "Unmanned aerial vehicle (UAV) for ecological research", PLoS One, Vol. 10, No. 9.

[7] Garg, R. G. (2016) "Insuring Indian farmers more effectively", ÏCT Update 'Drones for agriculture ', No. 82. [Online]. Available: http://ictupdate.cta.int/2016/04/21/insuring-indian-farmers-moreeffectively/ [Accessed: 15 Sept. 2018].

[8] Greenwood, F. (2016) "Drones on the horizon: new frontier in agricultural innovation", ICT Update 'Drones for agriculture', No. 82. [Online]. Available: http://ictupdate.cta.int/2016/04/21/drones-onthe-horizon-new-frontier-in-agricultural-innovation/ [Accessed: 15 Sept. 2018].

[9] Herwitz, S. R., Johnson, L. F., Dunagan, S. E., Higgins, R. G., Sullivand, D. V. and Zheng, J. (2004) "Imaging from an unmanned aerial vehicle: agricultural surveillance and decision support", Computers and Electronics in Agriculture, Vol. 44, No. 1, pp. 49-61. ISSN 0168-1699. DOI 10.1016/j.compag.2004.02.006.

[10] Kahan, D. G. (2007) "Farm management extension services: a review of global experience", Food and Agriculture Organization of the United Nations Rome.

[11] Laliberte, A. A., Rango, A and Slaughter, A. (2006) "Unmanned Aerial Vehicles (UAVs) for rangeland remote sensing ", In Proc. $3^{\text {rd }}$ Annual Symposium research Insights in Semiarid Ecosystems RISE, USDA-ARS Walnut Gulch Experimental Watershed.

[12] Liaghat, S and Balasundram, K. S. (2010) "A Review: The Role of Remote Sensing in Precision Agriculture", American Journal of Agricultural and Biological Sciences, Vol. 5, No. 1, pp. 50-55, ISSN 1557-4989. DOI 10.3844/ajabssp.2010.50.55.

[13] Nigon, T. J., Mulla, D. J., Rosen C. J., Cohen, Y., Alchanatis V., Knight J. and Rud, R. (2015) "Hyperspectral aerial imagery for detecting nitrogen stress in two potato cultivars", Computers and Electronics in Agriculture, Vol. 112, pp. 36-46. ISSN 0168-1699. DOI 10.1016/j.compag.2014.12.018.

[14] Oltmanns, S. D., Marzolff, I, Peter, K. D. and Ries, J. B. (2012) "Unmanned Aerial Vehicle (UAV) for Monitoring Soil Erosion in Morocco", Remote Sensing, Vol. 4, No. 11, pp. 3390-3416. ISSN 2072-4292. DOI 10.3390/rs4113390.

[15] Peña, J. M., Torres-Sánchez, J., De Castro, A. I., Kelly, M. and López-Granados, F. (2013) "Weed mapping in earlyseason maize fields using object-based analysis of unmanned aerial vehicle (UAV) images", PLoS One, Vol. 8, No. 10. ISSN 1932-6203. DOI 10.1371/journal.pone.0077151.

[16] Propeller Aero (2018) [Online]. Available: https://www.propelleraero.com/gsd-calculator/. [24 Oct 2018].

[17] Tahar, K. N. (2012) "A new approach on slope data acquisition using unmanned Aerial Vehicle", International Journal of Recent Research and Applied Studies, Vol. 13, No. 3. ISSN 2349-4891.

[18] Tellidis, I and Levin, E. (2014) "Photogrammetric Image Acquisition with Small Unmanned Aerial Systems", ASPRS 2014 Annual Conference, March 23-28, 2014.

[19] Yano, I. H., Alves, J. R., Santiago, W. E and Mederos, B. J. T. (2016) "Identification of weeds in sugarcane fields through images taken by UAV and Random Forest Classifier", IFAC-Papers On-Line ,Vol. 49-16, pp. 415-420. ISSN 2405-8963. DOI 10.1016/j.ifacol.2016.10.076.

[20] Zinke, P and Flener, C. (2013) "Experiences from the use of Unmanned Aerial Vehicles (UAV) for River Bathymetry Modeling in Norway", 'Technoport - Sharing possibilities' conference, Trondheim, Norway, on 16-18 April 2012.

[21] Xiang, H and Tian, L. (2011) "Development of a low-cost agricultural remote sensing system based on an autonomous unmanned aerial vehicle (UAV)", Bio-systems Engineering, Vol. 108, No. 2, pp. 174-190. ISSN 1537-5110. DOI 10.1016/j.biosystemseng.2010.11.010. 